\title{
Meditation and melotherapy as adjuvant treatments of chronic dermatoses
}

\author{
Bogdan M Tarcău, Ioana A Halip, Adriana I Pătrașcu, Laura Stătescu, \\ Dan Vâță, Adrian Cantemir, Laura Gheucă Solovăstru
}

\begin{abstract}
Bogdan Marian Tarcău - MD, Dermatology Clinic, "Saint Spiridon" County Hospital, Iasi, Romania

Ioana Alina Halip - MD, PhD, assistant professor, Department of Dermatology, "Grigore T. Popa" University of Medicine and Pharmacy, Faculty of Medicine, Iasi, Romania.

Adriana Ionela Pătrașcu - MD, Dermatology Clinic, "Saint Spiridon" County Hospital, Iasi, Romania

Laura Stătescu - MD, PhD, associate professor, Department of Dermatology, "Grigore T. Popa" University of Medicine and Pharmacy, Faculty of Medicine, Iasi, Romania. Dermatology Clinic, "Saint Spiridon" County Hospital

Dan Vâță - MD, PhD, associate professor, Department of Dermatology, "Grigore T. Popa" University of Medicine and Pharmacy, Faculty of Medicine, Iasi, Romania. Dermatology Clinic, "Saint Spiridon" County Hospital

Adrian Cantemir - MD, PhD, assistant professor, Department of Psychiatry, "Grigore T. Popa" University of Medicine and Pharmacy, Faculty of Medicine, Iasi, Romania

Laura Gheucă Solovăstru - MD, PhD, professor, Department of Dermatology, "Grigore T. Popa" University of Medicine and Pharmacy, Faculty of Medicine, Iasi, Romania. Dermatology Clinic, "Saint Spiridon" County Hospital
\end{abstract}

\section{ABSTRACT}

Stress levels have increased overall, moreover since the beginning of the coronavirus disease 2019 pandemic. Several chronic dermatoses, such as psoriasis and atopic dermatitis, are well known to be caused or aggravated by stress. It is common sense that, especially now, the patients affected by stress need to be offered more alternatives in order to control their skin conditions. Psychodermatology is a subspecialty of dermatology which through the joint efforts of dermatologists, psychiatrists and psychologists targets the complex mind-skin pathways. Meditation is a common arousal reduction intervention in the field of psychodermatology and it works by redirecting one's thoughts to calm one's mind. Melotherapy (music therapy) is another widely accesible stress reduction intervention, which despite its simplicity could prove efficient and address the general low compliance to psychological procedures.

\section{KEY WORDS:}

Psychodermatology, meditation, melotherapy, chronic dermatoses, psoriasis. 


\section{INTRODUCTION}

COVID-19 (coronavirus disease 2019) is the disease associated with the novel coronavirus 2 of the Severe Acute Respiratory Syndrome (SARS-CoV-2) $(1,2)$. The pandemic caused by this virus led to several safety measures such as home isolation, transport restrictions, social distancing and the use of protection equipment (3). All these measures, added to the fear factor of COVID-19, contribute to the psychological discomfort, stress and anxiety among the general population. This context represents the premise to an increased burden of the chronic dermatoses caused or aggravated by stress. Now, more than ever, psychodermatology must step forward through a mutidisciplinary team of dermatologists, psychiatrists and psychologists and help patients suffering from stress.

Psychodermatology is a relatively new subspecialty of dermatology which is gaining momentum. It aims at aiding in the treatment of cutaneous diseases by psychological and psychiatriac means which reduce the stress levels and control the harmful thoughts and behaviours. The effects of such interventions rely on the complex interactions between the nervous, immune, endocrine and cutaneous systems (4). Several psychological techniques can be used in the management of chronic dermatoses, such as: cognitive behavioural therapy, hypnosis, auto-hypnosis, meditation, guided imagery and melotherapy (music therapy) (5).

\section{METHODES}

This review of the literature will present the results of meditation and melotherapy recorded in the field of dermatology up until july 2021. The information presented includes evidences from peer-reviewed journals and controlled trials. Only articles written in English were reviewed.

\section{PSORIASIS AND STRESS}

Psoriasis is a chronic inflammatory skin condition, with a prevalence varying from $0.91 \%$ to $8.5 \%$ of the population worldwide (6). Genetic, epigenetic, environmental and lifestyle factors are associated with the onset and recurrence of psoriasis (7). The course of the disease is defined by periods of remissions and exacerbations (8).

Psychological stress does not only trigger exacerbations, but also contributes to the initial development of the disease in predisposed individuals (9-12). Fortune et al. studied the clearence of psoriasis during phototherapy, which was impaired in the patients who manifested excessive stress (13). The link between stress and psoriasis was also observed in the general population, as in two retrospective studies on 491 patients, stress was believed to be responsible for the appearance or exacerbation of the psoriasis in $37-88 \%$ of the patients $(14,15)$. Daily stressors cause increased itch and psoriasis severity, especially in the excessively worried individuals $(16,17)$.

Manolache et al. investigated the degree of stress in a targeted population and reported more stressful events in 169 patients with psoriasis than in the control group. In over half of these patients $(54 \%)$, at least one stressful event in the previous year was linked to the onset or recurrence of the disease (18).

A stress responder is defined as a psoriasis patient who deals with increased amounts of stress and holds the stress responsible for the worsening of his skin disease (19). It has been reported that high stress responders have a 
lower cortisol response to acute stress compared with low stress responders (20).

Psoriasis is still an incurable condition in which the physical burden is doubled by the psychological one. Stigma, social anxiety, negative self-image and higher levels of depressive symptoms are observed in the patients with psoriasis, especially when the lesions are distributed on more visible areas such as the arms, hands, head and neck (21). $62 \%$ of the psoriasis patients suffer from depression (22) and 43\% from anxiety (23).

The lack of adequate depression management is reflected in the fact that suicidal ideation is not uncommon, with reports of 1 out of 5 psoriasis patients with suicidal thoughts (24). The patients suffering from psoriasis are even more prone to commit suicide or to develop psychiatric disorders than the patients diagnosed with melanoma, one of the most aggressive cutaneous conditions (25). Psoriasis may also be wrongly considered contagious, contributing to the stigmatization (26).

\section{PSORIASIS AND MEDITATION}

Given the tremendous psychological impact psoriasis can have and the obvious link between stress and the disease, researchers have tested different approaches to reduce the arousal levels in the psoriasis patients.

Meditation has long been used for reducing stress and it originates from the eastern culture. Meditation can be practiced as mindfulness meditation, mantra meditation, yoga, tai chi and chi gong (27). Mindfulness meditation is the most researched method and it has been succesfully used in various mental and physical health conditions. It is typically described as non-judgemental attention to experiences in the present moment and it implies regulating one's attention and approaching one's experiences with openness and acceptance (28).

Several mindfulness-based stress reduction (MBSR) studies have shown a non-specific effect on stress reduction, similar to that observed with standard relaxation practices. Meditation could reduce the stress levels by improving self-regulation, promoting neuroplasticity and by increasing the parasympathetic activity which in turn interferes with the sympathetic-adrenalmedullary and hypothalamic-pituitary-adrenal axes (29-31).

Jon Kabatt-Zinn (the author of the mindfulness-based stress reduction programme), in a blinded randomized controlled trial from 1998, investigated the effect of meditation on psoriasis patients undergoing phototherapy. 37 patients with moderate to severe psoriasis were divided in 2 major groups - ultraviolet B (UVB) and psoralen plus ultraviolet A (PUVA), and subsequently each group was divided into control and meditation. The meditation groups had to listen to a recorded mindfulness meditation audiotape during 40 phototherapy sessions, while the control groups were instructed to relax by any means. The meditation group was instructed through the tape how to increase their moment-to-moment nonjudgmenetal awareness of breathing, body sensations, sounds, thoughts, feelings and they were asked to visualize how the ultraviolet light alone (UVB group) or in combination with psoralen (PUVA group) slowed down the growth and division of the skin cells. The group receiving the intervention through meditation reached skin clearing significantly faster (halfway point $\mathrm{p}=0.013$; clearing point $\mathrm{p}=0.033$ ). Neither group recorded any significant changes in the psychometric assessment (Symptom Checklist 90 Revised, SCL-90-R) or in the anxiety level 
\%

(State-Trait Anxiety Inventory, STAI) between pre-intervention and postintervention. Although the results are impressive, the patients have not been observed over a longer period of time (followup period of 1 week) and so the duration of remission was not assesed. Moreover, an attrition rate of $37.8 \%(n=14)$ was recorded (32).

Gaston et al. randomly assigned 18 psoriasis patients to 4 groups: meditation $(n=5)$, meditation and imagery $(\mathrm{n}=4)$, waiting list $(n=5)$ and treatment-free control group $(n=5)$. The meditation and imagery groups received the same meditation training during the first 6 weeks, while afterwards the patients in the imagery group were taught how to imagine their lesions transforming and healing. After a 12-week intervention (4 weeks pre- and postbaseline periods), the meditation group recorded a significantly better improvement in psoriasis severity, with no substantial benefits from the addition of imagery techniques. The authors reported a positive correlation between the psoriasis symptom severity and both psychological distress $(\mathrm{p}<0.01)$ and adverse life event impact $(\mathrm{p}<0.05)$. The limitations of this study consist in the reduced number of subjects, the lack of mean baseline disease severity quantification and the lack of psychological assessment. The attrition rate was $25.0 \%(\mathrm{n}=6)(33)$.

In a pilot study from 2015, Fordham et al. examined 29 psoriasis patients who were randomly distributed in either the mindfulness-based cognitive therapy (MBCT) group or the control group. The patients involved in the study were considered stress responders according to their evaluation through the Illness Perception Questionnaire (believed stress caused or exacerbated their psoriasis). The MBCT group received a 8week mindfulness group therapy intervention in addition to the usual psoriasis therapy, while the control group received only the psoriasis therapy. The MBCT group recorded statistically lower Self-administered Psoriasis Area Severity Index $(p=0.05)$ and Dermatology Life Quality Index $(\mathrm{p}=0.2)$ impairment scores than the control group. However, the reduction in stress and distress levels, evaluated through the Perceived Stress Scale $(\mathrm{p}=0.94)$ and Hospital Anxiety and Depression Scale $(p=0.11)$, was nonsignificant. The follow-up time was not described and the attrition rate was $45 \%$ $(n=10)$, patients dropping out of the study due to impracticality (34).

\section{PSORIASIS AND MUSIC THERAPY}

Lazaroff and Shimshoni investigated the effects of Medical Resonance Therapy Music on 28 neurodermatitis and 20 psoriasis patients, while the control groups consisted of 10 neurodermatitis and 10 psoriasis patients. Medical Resonance Therapy Music was developed by the classical composer and musicologist Peter Huebner in 1987. It is based on the ancient music medicine of Pythagorus, according to which the harmony laws of the world of music are identical to the harmony laws of the biological life. All the psoriasis patients received their psoriasis treatment over the course of 14 days. The patients in the experimental group were additionaly offered 3 x 30-minute sessions of listening to Medical Resonance Therapy Music everyday, while the patients in the control group were instructed to relax by any means. The degree of sickness was evaluated by the doctors according to a five-unit scale. After the intervention, at the end of the 14 days, the degree of sickness in the psoriasis experimental group was reduced by $65 \%$. The control group recorded a reduction in the degree of sickness of just $20 \%$. The stimulus to scratch had reduced in the experimental group by $86 \%$, while in the control group by 
29\%. The heart rate and the blood pressure had also lower values after the music therapy intervention (35).

\section{NEURODERMATITIS AND MUSIC THERAPY}

In the same study conducted by Lazaroff and Shimshoni on psoriasis patients, the effect of Medical Resonance Therapy Music was also tested on 28 neurodermatitis patients. After 14 days of 3 therapy music sessions a day, the degree of sickness was reduced by $41 \%$, versus $12 \%$ in the control group. The stimulus to scratch in the experimental group reduced by $59 \%$ versus $14 \%$ in the control group. Blood pressure and heart rate were also influenced, as lower values were recorded in the experimental group (35).

\section{ATOPIC DERMATITIS AND MEDITATION CONCLUSIONS}

Given the actual COVID-19 pandemic situation, arousal reduction techniques could play an even greater role in reducing the burden of stress-associated diseases. Mindfulness meditation has been investigated in several studies with promising results in psoriasis patients $(32,33,34)$, in contrast to atopic dermatitis patients (36). Melotherapy, however, was investigated in only one psoriasis and neurodermatitis study and even though it proved to be an efficient adjunctive therapy (more in psoriasis than in neurodermatitis), no statistical data was reported (35).

The studies investigating mindfulness meditation and music therapy share the same limitations as any other psychological intervention, such as the lack of a standardized protocol, high attrition rates and small sample sizes. This points to the need of more quality studies, comparing different psychological interventions and investigating which intervention best suits specific subsets of patients, in the effort to minimize the high dropout rates.

\section{ACKNOWLEDGEMENTS AND DISCLOSURES}

The authors declare that they have no conflicts of interest to disclose.

\section{REFERENCES}

1. WHO. Naming the coronavirus disease (COVID-19) and the virus that causes it. World Health Organization (WHO); https:// www. who.int/emergencies/ diseases/novel-coronavirus-2019/technical-guidance/naming-thecoronavirus-disease-(covid-2019)-and-the-virusthat-causes-it.

2. Coronaviridae Study Group of the International Committee on Taxonomy of Viruses. The species Severe acute respiratory syndrome-related coronavirus: classifying 2019-nCoV and naming it SARS-CoV-2. Nat Microbiol. 2020 Apr;5(4):536-544. doi: 10.1038/s41564-020-0695-z. 


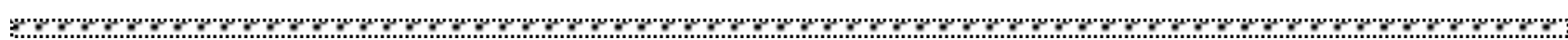
3. Cucinotta D, Vanelli M. WHO Declares COVID-19 a Pandemic. Acta Biomed. 2020 Mar 19;91(1):157-160. doi: 10.23750/abm.v91i1.9397

4. Jafferany M, Franca K. Psychodermatology: Basics Concepts. Acta Derm Venereol. 2016 Aug 23;96(217):35-7. doi: 10.2340/00015555-2378.

5. Shenefelt PD. Psychological interventions in the management of common skin conditions. Psychol Res Behav Manag. 2010;3:51-63. doi: 10.2147/prbm.s7072.

6. Ryan C, Korman NJ, Gelfand JM et al. Research gaps in psoriasis: opportunities for future studies. J Am Acad Dermatol 2014; 70:146-167.

7. Albanesi C, Madonna S, Gisondi P, Girolomoni G. The interplay between keratinocytes and immune cells in the pathogenesis of psoriasis. Front Immunol. 2018;9:1549.

8. Gaikwad R, Deshpande S, Raje S. et al. Evaluation of functional impairment in psoriasis. Indian J DermatolVenereol Leprol. 2006;72:37-40.

9. Heller MM, Lee ES, Koo JY. Stress as an influencing factor in psoriasis. Skin Therapy Lett. 2011;16:1-4.

10. Rousset L, Halioua B. Stress and psoriasis. Int J Dermatol.2018;57:1165-72.

11. Hunter HJ, Griffi ths CE, Kleyn CE. Does psychosocial stress play a role in the exacerbation of psoriasis? Br J Dermatol. 2013;169:965-74.

12. Malhotra SK, Mehta V. Role of stressful life events in induction or exacerbation of psoriasis and chronic urticaria. Indian J Dermatol Venereol Leprol. 2008;74:594-9.

13. Fortune DG, Richards HL, Kirby B, et al. Psychological distress impairs clearance of psoriasis in patients treated with photochemotherapy. Arch Dermatol. 2003;139:752-6.

14. Al'Abadie MS, Kent GG, Gawkrodger DJ. The relationship between stress and the onset and exacerbation of psoriasis and other skin conditions. Br J Dermatol. 1994;130:199-203.

15. Fortune DG, Richards HL, Main CJ, Griffiths CEM. What patients with psoriasis believe about their condition. J Am Acad Dermatol. 1998;39:196-201.

16. Verhoeven EW, Kraaimaat FW, de Jong EM, et al. Effect of daily stressors on psoriasis: a prospective study. J Invest Dermatol. 2009;129:2075-7.

17. Verhoeven EW, Kraaimaat FW, de Jong EM, et al. Individual differences in the effect of daily stressors on psoriasis: a prospective study. Br J Dermatol. 2009;161:295-9.

18. Manolache L, Petrescu-Seceleanu D, Benea V. Life events involvement in psoriasis onset/recurrence. Int J Dermatol. 2010;49:636-41.

19. Gupta MA, Gupta AK, Kirkby S, Schork NJ, Gorr SK, Ellis CN, Voorhees JJ. A psychocutaneous profile of psoriasis patients who are stress reactors. A study of 127 patients. Gen Hosp Psychiatry. 1989 May;11(3):166-73.

20. Richards, H., Ray, D., Kirby, B., et al. (2005), Response of the hypothalamic-pituitary-adrenal axis to psychological stress in patients with psoriasis. British Journal of Dermatology, 153: 1114-1120.

21. Lakuta P, Marcinkiewicz K, Bergler-Czop B, et al. Associations between site of skin lesions and depression, social anxiety, body-related emotions and feelings of stigmatization in psoriasis patients. Adv Dermatol Allergol.2018;35:606.

22. Esposito M, Saraceno R, Giunta A et al. An Italian study on psoriasis and depression. Dermatology 2006; 212: $123-127$.

23. Richards HL, Fortune DG, Griffiths CE, Main CJ. The contribution of perceptions of stigmatisation to disability in patients with psoriasis. J Psychosom Res 2001; 50: 11-15.

24. Krueger G, Koo J, Lebwohl M et al. The impact of psoriasis on quality of life: results of a 1998 National Psoriasis Foundation patientmembership survey. Arch Dermatol 2001; 137: 280-284.

25. Pompili M, Snast I, Reiter O, et al. Psychological stress and psoriasis: a systematic review and meta-analysis. BJD. 2018;178:1044-55.

26. Bhosle MJ, Kulkarni A, Feldman SR, Balkrishnan R. Quality of life in patients with psoriasis. Health Qual Life Outcomes 2006; 4: 35 .

27. Ospina, M. B. et al. Meditation practices for health: state of the research. Evid. Rep. Technol. Assess. (Full Rep.) 155, 1-263 (2007).

28. Baer, R. A. Mindfulness training as a clinical intervention: a conceptual and empirical review. Clin. Psychol. Sci. Practice 10, 125-143 (2003).

29. Thayer, J. F. \& Lane, R. D. A model of neurovisceral integration in emotion regulation and dysregulation. J. Affective Disord. 61, 201-216 (2000). 

30. Creswell, J. D. in Handbook of Mindfulness: Theory, Research, and Practice Ch. 23 (eds Brown, K. W., Creswell, J. D. \& Ryan, R. M.) (Guildford Press, 2014).

31. Ditto, B., Eclache, M. \& Goldman, N. Short-term autonomic and cardiovascular effects of mindfulness body scan meditation. Ann. Behav. Med. 32, 227-234 (2006).

32. Kabat-Zinn J. Influence of a mindfulness meditation-based stress reduction intervention on rates of skin clearing in patients with moderate to severe psoriasis undergoing phototherapy (UVB) and photochemotherapy (PUVA). Psychosom Med. 1998;60:625-632.

33. Gaston L, Crombez J-C, Joly J, Hodgins S, Dumont M. Efficacy of Imagery and Meditation Techniques in Treating Psoriasis. Imagination, Cognition and Personality. 1989;8(1):25-38. doi:10.2190/PQKE-CMAM-T4MTQVBL

34. Fordham, B., Griffiths, C. E. M., \& Bundy, C. (2014). A pilot study examining mindfulness-based cognitive therapy in psoriasis. Psychology, Health \& Medicine, 20(1), 121-127. doi:10.1080/13548506.2014.902483

35. Lazaroff I, Shimshoni R. Effects of Medical Resonance Therapy Music on patients with unipsoriasis and neurodermatitis--a pilot study. Integr Physiol Behav Sci. 2000 Jul-Sep;35(3):189-98. doi: 10.1007/BF02688779.

36. Bostoen J, Bracke S, De Keyser S, Lambert J. An educational programme for patients with psoriasis and atopic dermatitis: a prospective randomized controlled trial. Br J Dermatol. 2012 Nov;167(5):1025-31. doi: 10.1111/j.13652133.2012.11113.x.

\section{Correspondence:}

Bogdan Marian Tarcău,

MD, Dermatology Clinic, “Saint Spiridon” County Hospital, Bd. Independentei, nr. 1, Iasi, Romania, bogdan.tarcau@yahoo.com

Submission: 14 jul 2021

Acceptance: 28 aug 2021 